\title{
A Review of Negative Polarity Item Studies*
}

\author{
Xiangyan Wu \\ Foreign Language School \\ Linyi University \\ Linyi, China
}

\begin{abstract}
Negative Polarity Item refers to some language structures which are limited by negative contexts. It could be found in many languages and applied in different parts within a sentence. Since 1980s, Negative Polarity Item has attracted wide attentions from home and abroad, which includes research on Negative Polarity Item's syntax, semantics, pragmatics and etc. By analyzing the studies of Negative Polarity Item from the past 6 decades, this article finds that there is still debate on its concept, category, licensing mechanism, pragmatics functions, while deeper and fully researched studies are expected to be done in the future.
\end{abstract} review

Keywords-Negative Polarity Item; Polarity Sensitivity;

\section{INTRODUCTION}

Negative Polarity Item (NPI) is a special language sensitivity phenomenon with obvious features of forms and distribution. There is no common statement of its definition and category yet. What type of structure can be called NPI? Besides some adverbial NPI such as "si hao (丝毫)", "cong lai (从来)", is there any other kinds of NPI? And what features do they have? These questions would be helpful to identify the syntax and semantic features of Negative Polarity. In addition, as a part a negative category, the findings of these studies could enrich the negative theory which leads to the development of linguistics.

\section{The Definition OF THE CONCEPT OF NEGATIVE POLARITY ITEM}

The study of NPI is originally from the Western, started by Klima's specific research on Polarity Sensitivity in 1964. The Western linguistics divided this phenomenon into two terms which are Positive Polarity Item and Negative Polarity Item, for instance, 'some' and 'any' in English. However, when it comes to the concept of polarity item, scholars and researchers have not reached the same stage. Baker (1970) pointed that words or phrases that can only be used in positive context are Positive Polarity Items, while words and phrases that can only be used in negative context are Negative Polarity Items. While different views have given. Some suggest that part of NPI can be applied in rhetorical sentences, comparative sentences and conditional sentences.

* This research was supported by the Scientific Research Starting Foundation of Linyi University for Teachers with Doctor's Degrees in 2016.

\author{
Lei Wang** \\ The Guidance Center of Employment \\ Linyi University \\ Linyi, China \\ **Corresponding Author
}

Therefore, the above statement is not valid.

Although details vary from person to person, we still figure out that the concept of polarity item is generally defined by distribution and degree by most scholars (Ladusaw 1979, 1992, Linebarger 1987, Zwarts 1990, 1993, 1995, Lu 1986, An 1991, Shi 2001, Cheng 2007). Summarizing from the related research and corpus, NPI could mean words or phrases which co-exist with Negative Operators in negative sentences mostly, and weaken or strengthen the negative degree of its scope.

\section{LITERATURE REVIEW}

In Western countries, NPI studies can be traced back to about 60 years ago, while there is only 40 years' research history in China. Generally, compared to other language phenomena, specialized NPI research is rarely to see and sometimes is included in other negative structures studies. Hence, this field still lacks of professionalism. For example, there were no more than 10 published research papers each year before 2009 in China, after that there are just 20 published articles every year based on corpus and computer technology development.

Except starting time, angles are set differently in NPI research. Chinese scholars tend to analysis this phenomena from the aspects of licensing mechanism, pragmatic function and bi-directional association, while foreign research is more about syntax and semantic analytic.

Syntax analysis is a common research method in the early age since many scholars looked into NPI from the aspect of syntax. Kilma (1964) believed that a sentence containing NPI is grammatical, if and only if the NPI is in the domain of negation operator. With more thorough research of language phenomena, researchers led by Fauconnier (1975) began to carry out NPI studies based on semantic level. Fauconnier (1975) suggested only at a relative level could we correctly explained the meaning of the polarity item. According to this, Ladusaw (1979) proposed entailment relationship which is promoted by Chierchia (1990). Chierchia (1990) pointed that downward monotonicity is a necessary condition to constitute negative context. Monotonicity has been a controversial issue as some opposites like Linebarger $(1980,1987)$ insisted that even some contexts made the NPI conform to the relevant rules of grammar, but they did not necessarily have the 
characteristics of downward monotonicity or downward implication.

Domestically, Lv (1980) advised that in Chinese there was a class of words or sentences that could only appear in negative sentences.' when he analyzed the meaning of 'ye (也)', but the concrete definition of NPI was not discussed in his essay. Korean professor of the Chinese Department Heo Seong Do introduced Negative Polarity into Chinese with description of non-defining NPI in 1984. Jiaxuan Shen proposed the concept of Polarity Words and analyzed it by hypothetic markedness pattern in 1999, which means some words in language could only be used in affirmative sentences, and some words could only be used in negative sentences. If affirmation and negation are regarded as positive and negative poles, some words in the language belong to polarity words.

From 1990s to the early 21th century, most related research was about summarizing adverbial NPI catalogue (An 1991, Zheng 1996, Shi 2001). Rupan A listed some negative adverbs, verbs, adjectives and nouns like 'chi chi (迟迟), duan duan (断断), duan ran (断然), jie ran (截然), jiong ran (迥然), quan ran (全然), ya gen er (压根儿), si huo (死活), gen ben (根本)'. Jianping Zheng summed up 13 adverbial NPI which are 'bing (并), chi chi (迟迟), cong (从), duan (断), hao (毫), jue (决), jue (绝), si hao (丝毫), wan (万), wan wan (万万), ya gen er (压根儿), zai ye (再也)'. Moreover, he also added that adverbial NPI only takes up to $3 \%$ in Chinese, meanwhile there are other types of NPI. However, both of them did not have a fully discussion of this topic. Yuzhi Shi made a systematic study of NPI and listed 5 adverbial NPI which are 'jue (绝), hao (毫), duan (断), hao fa (毫发), ya gen er (压根儿)', then the distribution features of NPI as 'words with minimal semantic meaning could only be used in negative structures.' was summarized based on that.

Since the 21th century, NPI research expands from concept and catalogue to semantics, syntax and pragmatics in China. Sun (2005) calculated 30 adverbial negative polarity items in the early stage and divided them into modal adverbial NPI, time adverbial NPI, degree adverbial NPI and scope adverbial NPI. Those words are 'chi chi (迟迟), cong (从), cong lai (从来), bing (并), ya gen er (压根儿), gen ben (根 本), jue (绝), hao (毫), si hao (丝毫), hao fa (毫发), wan (万), wan wan (万万), duan (断), duan duan (断断), duan ran (断然), duan hu (断乎), duan duan hu (断断乎), quan ran (全然), si huo (死活), shi zhong (始终), zai ye (再也), qian wan (千万), jue ji (决计), qie (切), qie qie (切切), liao (了), gai (概), yi gai (一概)'. Chen and Luo (2005) advised that 'the semanteme of negative term was restricted by many factors, especially easy to be restricted by grammatical factors and grammatical levels, and the three interface models of syntax, semantics and pragmatics seemed to be related to the NPI.' Yin (2008) discussed NPI's concept and Licensing Mechanism in the syntax study of negatives and adverbs' co-occurrence. What should be noted here is the study of 'adverbs + no' structure, which is helpful to the origin and development research of adverbial NPI to some extent. In addition, weakening NPI can be applied as litotes (Jiang 2009).
In the recent years, comparison studies of Chinese NPI and other language's NPI have showed up. English-Chinese comparison studies take the most percentage among those (Song 2011, Zhang 2012). Meanwhile Japanese-Chinese and Korean-Chinese comparison are also included (Wang 2011, Gao 2012, Xiang 2011, Huang 2012 and Dong 2015). Mingyu Zhang (2012) carried out a systematical study of Polarity Sensitivity and Licensing Mechanism between Chinese and English based on a large amount of corpus. Moreover, Youyou Xiang (2011) made a Chinese-Korean comparison study in some NPI. Xingying Huang(2012) compared the NPI in Korean and Chinese thoroughly according to corpus. However, due to the limitation of corpus and overestimated the theory of downward-entailing, so some results are needed to be checked. While Lijing Dong (2015) selected 'ren he' in Chinese and 'A mu' in Korean as research objects.

There is a growing tendency of analyzing one specific NPI. Changhong Sui and Zhenyan Hou (2000) made a pragmatic research about adverbial NPI 'gen ben'. Dongxia Zhou and Wanmei Huang (2011) summarized the syntax, semantic and pragmatic function of 'yi gai (一概)'. Yuhui Lv (2005) and Li Zhang (2010) analyzed the phrase 'si hao (丝 毫)' in terms of syntax, and had a contrast structure study of 'si hao (丝毫) + bu (不) +verb' and 'si hao (丝毫) + mei you (没有) + verb'. Meanwhile, Xiezhong Jiang (2010) described the history of 'qian wan (千万)', then concluded that it is transformed from numerals and mainly applied in negative imperative sentences. Similarly, Chuntian Feng (2005) analyzed the development of 'cong (从)' from the history perspective. Qian Li (2006), Yong $\mathrm{Hu}$ (2006) and Shilin Ding (2009) all had a study about the word 'bing (并)', while Li (2006) pointed that presupposition is the most important pragmatic function of it. In addition, there is a certain number of research which insists that the co-existence of polarity items and 'dou (都)' is unique in Chinese. Yulin Yuan (2007) held the view that 'dou (都)' applies the concealed negation, while Cheng \& Giannakidou (2013) and Weiping Wen (2013) believed that definiteness of polarity item could be offered by 'dou (都)', Yuzhi Shi (2004) and Yong Jiang (2015) pointed that 'dou (都)' stands for the denotation meaning of polarity item. And Haihua Pan (2017) proposed that the co-occurrence of 'dou (都)' and 'polarity item' is not only a challenge to the theory of universal polarity in Chinese, but a strong support for DE's promise.

Based on the above research theories, some scholars turn their filed into the acquisition of NPI. It is mainly focused on the acquisition order and condition among adults (Janssen 2008, Wenting Zhou 2007, Lina Tong 2008) and children (O’ Leary \& Crain 1994, Koster \& S. Van der Wal 1995, Tieu 2009, Yuming Li \& Qianrui Chen 1998, Zhou 2010).

\section{CONCLUSION}

By analyzing those previous studies, it can be concluded that there has been some theories and practical research about NPI worldwide. Yet, it is still necessary to improve related research, compared with a huge amount of negative category studies. For instance, present research is rarely concentrated on other fields except adverbial NPI. Also, in 
terms of research methods, the most applied one is downward-entailment theory while is still hated debated abroad. Hence, deeper research of NPI is essential.

\section{REFERENCES}

[1] Baker, C. Leroy. Double Negatives[J], Linguistic Inguiry1(2), 1970, pp.169-186.

[2] Changhong Sui \& Zhenyan Hou. The Pragmatics Analysis of Cardinally Negative Adverb[J], Journal of Linyi Teachers' College(5), 2000, pp.17-19.

[3] Chierchia G.. Meaning and Grammar: an introduction to semantics[M], University of New England Press, 1990.

[4] Chuntian Feng. Historical Investigation on the Adverbs of "cong" (从) \&"fou ( 否 )" Categories[J], Linguistic Research(4), 2005, pp.36-38.

[5] Dongxia Zhou \& Wanmei Huang. Multidimensional investigation of modern Chinese Adverb "Yigai"[J], Time Education (1), 2011, pp.268-269.

[6] Fauconnier, G. Pragmatic Scale and Logical Structure[J], Linguistic Inquiry 6 (3), 1975, pp.353-375.

[7] Hongbo Yin. a syntactic and semantic study of the conjunction of negatives and adverbs[D], Doctoral Dissertation of the Graduate School of Chinese Academy of social sciences, 2008

[8] Jia Xu Shen. Asymmetry and Markedness Theory[M], commercial press, 2015

[9] Jianming Lu. Universal Subject Sentence and Other[J], Chinese language(3), 1986, pp.161-167.

[10] Jinji Song, a Contrastive Study of Negative Polarity Item between English and Chinese[D], Master's postgraduate thesis of Yanbian University, 2011.

[11] Kai Shek Chiang, The Historic Evolution of the Usage of Adverb "qianwan"[J], Journal of Qinzhou University(1), 2010, pp.71-74.

[12] Klima, E., Negation in English. In Jerry Fedor\&amp; Jerrold Kats.(Eds.) The Structure of Language[C], Engle wood Cliffs: Prentice-Hall, 1964.

[13] Klima, Edward, S., Negation in English[A]. In Fodor, J. A. and J. J. Katz (eds.), The Structure of Language[C], 1964, pp.246 -323.

[14] Ladusaw, William A., Polarity Sensitivity as Inherent Scope Relations[D], Ph.D. Dissertation, University of Texas, 1979.

[15] Ladusaw, William. A., Expressing Negation[A], In Proceedings of Semantics and Linguistics Theory II[C], 1992, pp.237-260

[16] Lijing Dong, A Contrastive Study of Negative Polarity Item between Korean 'A-MU' and Chinese 'Renhe'[D], Master's postgraduate thesis of Yanbian University, 2015.

[17] Linebarger, M., Negative Polarity and Grammatical Representation[J], Linguistics and Philosopby10(3), 1987, pp.325-387.

[18] Ning Gao, The cause of formation of Recessive Negatives in Chinese and Japanese: Reflecting on SHI Yuzhi's theory[J], foreign language teaching (1), 2012, pp.7-15.

[19] Qian Li, syntactic and pragmatic analysis of Modal Adverb "bing"[J], Modern Chinese(4), 2006, pp.75-77.

[20] Qin Sun, Investigation of denying special-purpose adverbial word of structure of modern Chinese[D], Master's postgraduate thesis of Guangxi Normal University, 2005.

[21] Rupan An, talked about the words which are often used in negative terms[J], Journal of Beijing Normal University (Social Sciences)(4), 1991, pp.52-56, p.109.

[22] Seong Do Heo, A Study on Adverb in Negation of Mandarin Chinese[J], Chinese Literature(12), The Society For Chinese Language And Literature, 1984, pp.189-210.

[23] Shilin Ding, a brief discussion on the relationship between the phonetic symbols and the phonetic sound[J], Modern Chinese(6), 2009, pp.141-142.

[24] Wenhua Cheng, On the Explanation of Negative Polarity Terms[J], Journal of Hefei University (Social Sciences)(4), 2007, pp.74-76
[25] Xiaoxiang Chen, Qiongpeng Luo, extreme sensitivity and promise conditions of negative words $[\mathrm{J}]$, Foreign Languages and Their Teaching(4), 2005, pp.8-13.

[26] Xin Wang, the Cognitive Semantic Analysis of "bu(不)" and "meiyou(没有)"[J], Language Teaching and Linguistic Studies(4), 2007, pp.26-33.

[27] Xingying Huang, A contrastive study of Negative Polarity Items in Chinese and Korean, Master's postgraduate thesis of Korea University, 2013.

[28] Yong Jiang\& Keyi Zhu, the Litotic use of Negative Polarity Items and Pragmatic Adjustment[J], foreign language teaching and Research (6), 2009, pp.410-416.

[29] Youyou Xiang, A contrastive study of Negative Polarity Items in Chinese and Korean[D], Master's postgraduate thesis of Busan University, 2011.

[30] Yuhui Lv, The Semantic Meaning of "the Slightest Amount" [J], Journal of Henan University (Social Sciences) (6), 2005, pp.125-127.

[31] Yuzhi Shi, Symmetry and Asymmetry between Affirmation and Negation (2nd Edition)[M], Beijing language and Culture University Press, 2001.

[32] Zwarts, F., The Syntax and Semantics of Negative Polarity, in S.Busemann-ed. Views on the Syntax-Semantics Interface II, Berlin, 1990

[33] Zwarts, F., Three Types of Polarity[J], Springer Netherlands(69), 1998, pp.177-238. 